\title{
A Role for Angiogenesis in Canine Cutaneous Histiocytoma Regression: Insights into an Old Clinical Enigma
}

\author{
DIANA COSTA ${ }^{1 *}$, RITA FERREIRA $^{1 *}$, JUSTINA PRADA $^{2,3}$, FELISBINA LUISA QUEIROGA $^{2,4,5}$, \\ PAULA RODRIGUES ${ }^{2,3}$, FILIPE SILVA $^{2,3}$ and ISABEL PIRES ${ }^{2,3}$ \\ ${ }^{1}$ Department of Genetics and Biotechnology, University of Trás-os-Montes and Alto Douro, Vila Real, Portugal; \\ ${ }^{2}$ Department of Veterinary Sciences, University of Trás-os-Montes and Alto Douro, Vila Real, Portugal; \\ ${ }^{3}$ Animal and Veterinary Research Centre (CECAV), \\ University of Trás-os-Montes and Alto Douro, Vila Real, Portugal; \\ ${ }^{4}$ Center for Research and Technology of Agro-Environment and Biological Sciences (CITAB), \\ University of Trás-os-Montes and Alto Douro, Vila Real, Portugal; \\ ${ }^{5}$ Center for the Study of Animal Sciences, CECA-ICETA, University of Porto, Porto, Portugal
}

\begin{abstract}
Background/Aim: Canine Cutaneous Histiocytoma $(\mathrm{CCH})$ is a Langerhans' cells benign tumour that undergoes spontaneous regression. The aim of the present study was to investigate the role of angiogenesis, a key step for tumour development, in $\mathrm{CCH}$ regression. Materials and Methods: 50 $\mathrm{CCH}$ samples were classified into 4 histological groups according to a regression scale, and evaluated for expression of vascular endothelial factor-A (VEGF-A) and its receptor VEGFR-2 as well as microvessel density (MVD). Results: Tumours during early stages of the regressive process had a lower MVD compared to later stages, while CCH tumoural cells showed a limited production of VEGF, but higher levels of VEGFR-2. On the contrary, tumours in advanced phases of regression showed a higher number of neovessels, probably associated with the inflammatory state and the healing process. Conclusion: Our results suggest that angiogenesis may be compromised at early stages of histiocytoma development and this may be a determinant of regression in this tumour.
\end{abstract}

Canine cutaneous histiocytoma (CCH) is a benign tumour on the dog's skin that, in almost all cases, undergoes spontaneous regression. It can occur anywhere on the dog's skin surface as

This article is freely accessible online.

*The Authors contributed equally to this article.

Correspondence to: Felisbina Queiroga, Department of Veterinary Sciences, University of Trás-os-Montes and Alto Douro, Quinta dos Prados, 5000-801 Vila Real, Portugal. Tel: +351 917826982, Fax: +351 259350480, e-mail: fqueirog@utad.pt

Key Words: Canine cutaneous histiocytoma, angiogenesis, VEGF, CD31. a result of neoplastic proliferation of epidermal dendritic cells, called the Langerhans cells. It is classified as a tumour of round cells, and is reported as the most frequent cutaneous neoplasm in dogs (1-3). CCH has similarities to some forms of human Langerhans cell histiocytosis ( $\mathrm{LCH})$, nevertheless, LCH has distinct clinical behaviour between $\operatorname{dog}$ s and humans (4). The regression phenomenon, typical of this disease, makes $\mathrm{CCH}$ an attractive system for the study of human Langerhans cell histiocytosis and has been considered as an unique model to understand the pathogeny of this enigmatic human disease (5-7).

Spontaneous tumour regression has been described for different types in both humans and animals, and is possibly associated with an efficient immune response (8-10). Several pathways are proposed for $\mathrm{CCH}$ regression namely the loss of the adhesion molecule E-cadherin, rise of Th1 proinflammatory cytokines such as IL-2, tumour necrosis factor$\alpha$ (TNF- $\alpha)$, interferon- $\gamma($ IFN- $\gamma)$, up-regulation of the nitric oxide synthase (iNOS) expression, the enzyme that produces the cytotoxic metabolite nitric oxide, and changes in the expression of extracellular matrix metalloproteinases, such as MMP-9 (6, 11-13). Major Histocompatibility Complex MHC class II is also related to tumour regression, the migration of MHC molecules in the cell periphery is a significant factor for the regression in $\mathrm{CCH}$ (4). Also, an imbalance between cell proliferation and apoptotic cell death, rather than a decrease in cell proliferation or an increase of apoptosis alone, is a driving force leading to CHH regression (10). Despite all this data, regression mechanisms are not yet fully understood.

Angiogenesis, as defined by the occurrence of neovessels and estimated by the microvessel density, is a crucial process to the growth, development and metastasis of several tumours in mammals (14). Either normal angiogenesis or 
tumoural angiogenesis are controlled by growth factors and their respective receptors. The vascular endothelial growth factor (VEGF-A) and its receptor VEGFR-2 (FIK-1) are amongst the most studied molecules that have been related with increased angiogenesis in several cancer types in humans and animals $(15,16)$.

Considering the high cellularity and proliferation rate of the histiocytoma, as well as the levels of apoptosis and necrosis usually observed (10), the aim of our work was to determine if a lack of an appropriated vascular supply, due to an insufficient production of VEGF-A, VEGFR-2 or poor microvessel formation could be associated with its spontaneous regression.

\section{Materials and Methods}

Tissue samples. Fifty $\mathrm{CCHs}$ were obtained from the archive of the Histopathology Laboratory of the University of Trás-os-Montes and Alto Douro (UTAD), Vila Real, Portugal. Paraffin sections of skin tumours samples were sectioned at $3 \mu \mathrm{m}$, and stained with Hematoxylin and Eosin (H\&E). The diagnosis and classification of histiocytomas were performed by two independent observers according to the world health organization (WHO) criteria. All histological preparations were observed systematically, according to the degree and location of lymphocytic infiltration being allocated to one of four groups representing different stages of tumour regression, according to previous studies $(6,17)$. Briefly, i) in group I, lymphoid inflammatory infiltration was absent or minimal in periphery, ii) in group II it was moderate, peripheral, and with formation of nodular structures, iii) in group III it was abundant both in the periphery and the centre of the tumour, and iv) in group IV it was diffuse, extending to the epidermal surface. Ulceration (absent, present), necrosis (absent, present), stroma (scarce, moderate, abundant), mitotic count ( $<22,2-5$ and $>5$ in high power field; $400 x$; Nikon ${ }^{\circledR}$ Eclipse E600 microscope, Nikon Instruments Inc., Melville, New York, USA), counting at least 10 fields) were also evaluated.

Immunohistochemical study. Immunohistochemistry was performed using the indirect staining method of Streptavidin-BiotinPeroxidase, using the UltraVision Detection System, HRP, (Thermo Fisher Scientific ${ }^{\circledR}$, Runcorn, Cheshire, UK). As a method of antigen recovery, the histological preparations were subjected to a microwave thermal pre-treatment, which involved three 5-min cycles in the microwave at 750 Watts in citrate buffer $(\mathrm{pH}=6.0 \pm 0.2)$. The antibodies used were VEGF (Ab-3 JH121, NeoMarkers ${ }^{\circledR}$, Fremont, California, USA; 1:100) and its receptor Flk-1 (A-3, Santa Cruz Biotechnology ${ }^{\circledR}$; California, USA, 1:100), and CD31 (Clone JC70A, Dako®, Glostrup, Denmark; 1:20). All incubations were made in a humid chamber overnight at $4^{\circ} \mathrm{C}$. The reaction sites were developed with the addition of a 3,3'-diaminobenzidine tetrahydrochloride (DAB) solution (Thermo Fisher Scientific ${ }^{\circledR}$, Runcorn, Cheshire, UK). The sections were immersed in a solution of Gill's Hematoxylin, dehydrated, cleared and mounted with Entellan synthetic resin $\left(\right.$ Merck $^{\circledR}$ Merck KGaA, Darmstadt, Germany). In each set of samples for each procedure, a positive control (canine angiosarcoma or breast carcinoma) and a negative control (an HCC sample in which the incubation of the primary antibody was performed using PBS only).
Evaluation of immunoreactivity. In order to evaluate the VEGF-A and VEGFR-2 (Flk-1) expression, a semi-quantitative analysis was performed according to the percentage $(\%)$ of positively-stained tumour cells: i) $0-5 \%$ as (negative), ii) $5-25 \%$ as + (focal), iii) $25-$ $50 \%$ as ++ (multifocal), iv) $>51 \%$ as +++ (diffuse). The intensity of marking was also evaluated: i) weak $(+)$, ii) moderate $(++)$, iv) strong $(+++)$. In the other hand, to determine the microvessel density, the counts were performed in the central region of the tumour, in order to avoid the ulcerated and necrotic regions, without prior knowledge of the sample's tumour group. From each histological preparation, two hotspots were identified under lowpower magnification $(40 \times)$. Then, under high power $(200 \times), 3$ fields were randomly selected in each selected area. Images were captured using a Nikon Eclipse E600 microscope with a Nikon DXM1200 digital camera (Nikon Instruments Inc., Melville, New York, USA) from each of these fields in JPEG format, at a 200× magnification (surface area $=28.96 \mathrm{~cm}^{2}$ ). The number of new vessels was subsequently digitally counted. Positive cells or cell aggregates that were clearly separated from adjacent neovessels, tumour cells and connective tissue elements were considered as individual neovessels. The average number of new vessels was calculated and expressed per 200` field.

Statistical analysis. The statistical analysis of the data was performed using the IBM ${ }^{\circledR}$ SPSS ${ }^{\circledR}$ Statistics program (Version 24.0, Version 24.0. Armonk, NY, USA). A descriptive analysis of categorical variables was performed, obtaining the absolute and relative frequencies of the categories under study. The microvessel density values were described according to the mean, median, standard deviation, minimum and maximum values. In order to evaluate the association between VEGF and its receptor with the histological groups, the chi-square test was performed. The associations between the microvessel density measured by CD31 and the histological group were determined by the Kruskal-Wallis non-parametric test, after checking the nonnormal distribution of the DMV values using the KolmogorovSmirnov test. When the microvessel density values were different between the categories, the Bonferroni test was performed to find out between which categories the differences were statistically significant. Associations were considered significant for values of $p<0.05$.

\section{Results}

Histopathological evaluation. Tumours formed nodular formations, exophytic proliferations, or "button"-shaped lesions. All lesions were unique, alopecic and often ulcerated. Neoplastic cells have an oval, round or reniform nucleus, usually eccentric, with an evident nucleolus and acidophilic cytoplasm that varies from moderate to abundant (17). Ulceration of the epidermis was present in 38 cases (76\%). Mitosis figures ranged from 0 to 5 in the high-power field: 11 tumours $(22 \%)$ had a mitosis count (MC) of less than 2, 20 cases had an MC between 2 and 5, and 19 cases (38\%) had an MC greater than 5. The tumour stroma was generally scarce $(n=38 ; 76 \%)$, while its abundance was only verified in 2 cases, being moderate in the others $(n=10)$. In more than half of the cases $(n=34 ; 63.3 \%)$ necrosis was not 


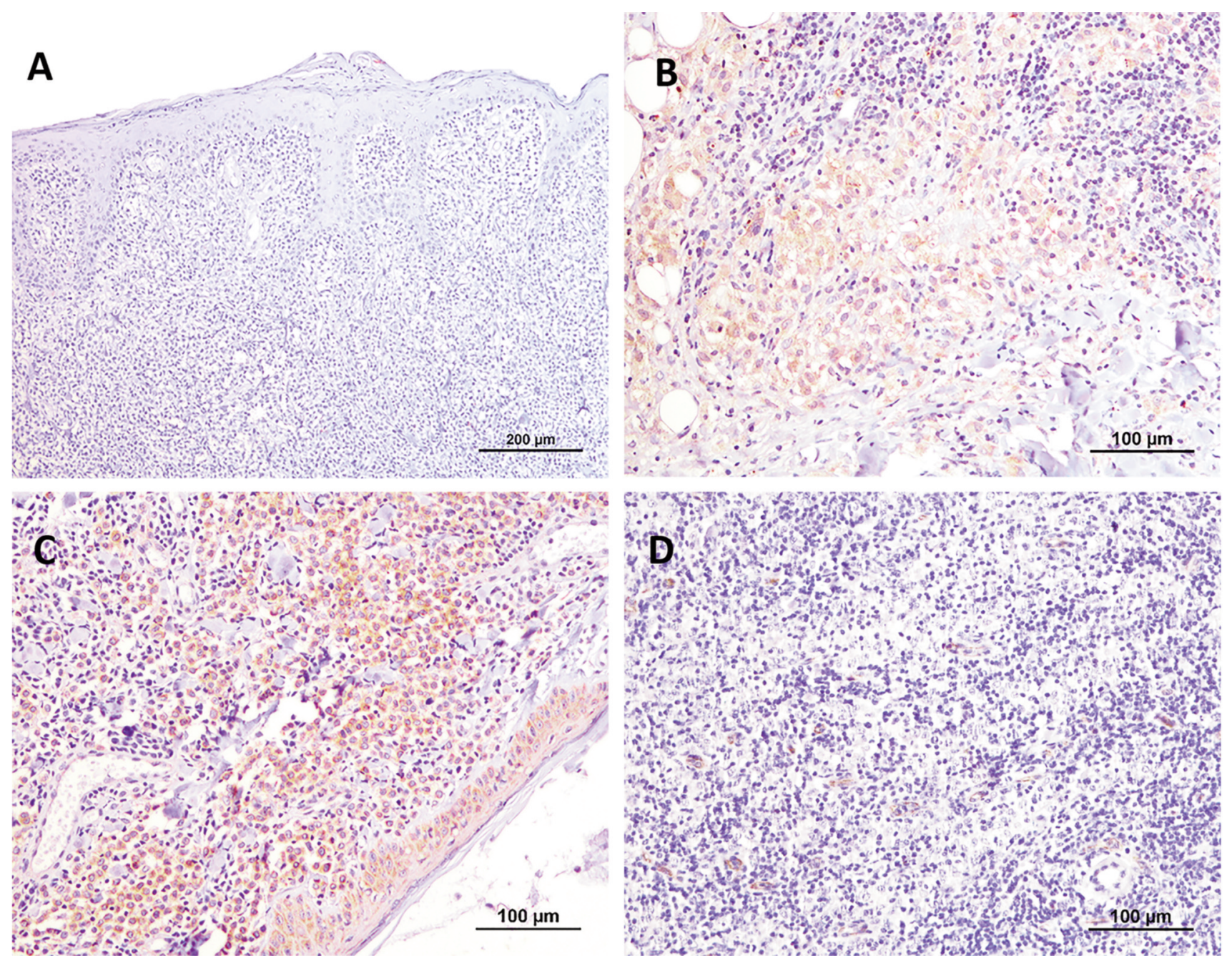

Figure 1. VEGF-A expression. A) Absence of VEGF-A immunoreactivity in a group I canine cutaneous histiocytoma; B) VEGF-A positive cells with a diffuse and strong labelling in a group IV canine cutaneous histiocytoma. C) VEGFR-2 positive cells with a diffuse and strong labelling in a group I canine cutaneous histiocytoma with a diffuse and strong labelling. D) CD31 staining of endothelial cells in a group IV canine cutaneous histiocytoma.

observed. Of all, $18 \mathrm{CCHs}$ were classified as group I (36\%), 13 as group II (26\%), 12 as group III (24\%), and 7 as group IV $(14 \%)$.

Expression of VEGF-A. Expression of VEGF-A was detected in the cytoplasm of tumour cells with a granular or diffuse pattern, as well as in macrophages (positive internal control). Immunoreactivity was not uniform throughout the preparation, with a greater expression in peripheral tumour cells of the deeper skin layer. Regarding the percentage of VEGF-A positive cells, most tumours were negative $(n=31$, $62 \%)$ or had focal positivity $(\mathrm{n}=6,12 \%)$ and 7 cases $(14 \%)$ presented diffuse expression. About the intensity of the immunostaining, $14 \%$ of the cases $(n=7)$ showed a strong reaction, 2 cases $(4 \%)$ had a low intensity of marking, and
10 cases (20\%) had a moderate intensity (Figure 1A and 1B).

A statistically significant association was observed between the percentage of VEGF-A positive cells and abundant tumour stroma $(p=0.039)$, and the presence of necrosis $(p=0.014)$. Thus, sparse stromal tumours were mostly negative for VEGF-A, while moderate stromal tumours were positive in more than half of the cases. Most tumours with evident necrotic areas did not express VEGFA. Labelling intensity showed a statistically significant association with necrosis $(p=0.016)$, similar to that was described for the extent of VEGF-A labelling.

VEGF-A expression was significantly associated with the tumour group [both for the percentage of VEGF-A positive cells $(p=0.002)$ and VEGF-A intensity of immunolabelling $p=0.009)]$. VEGF-negative tumours generally belonged to 


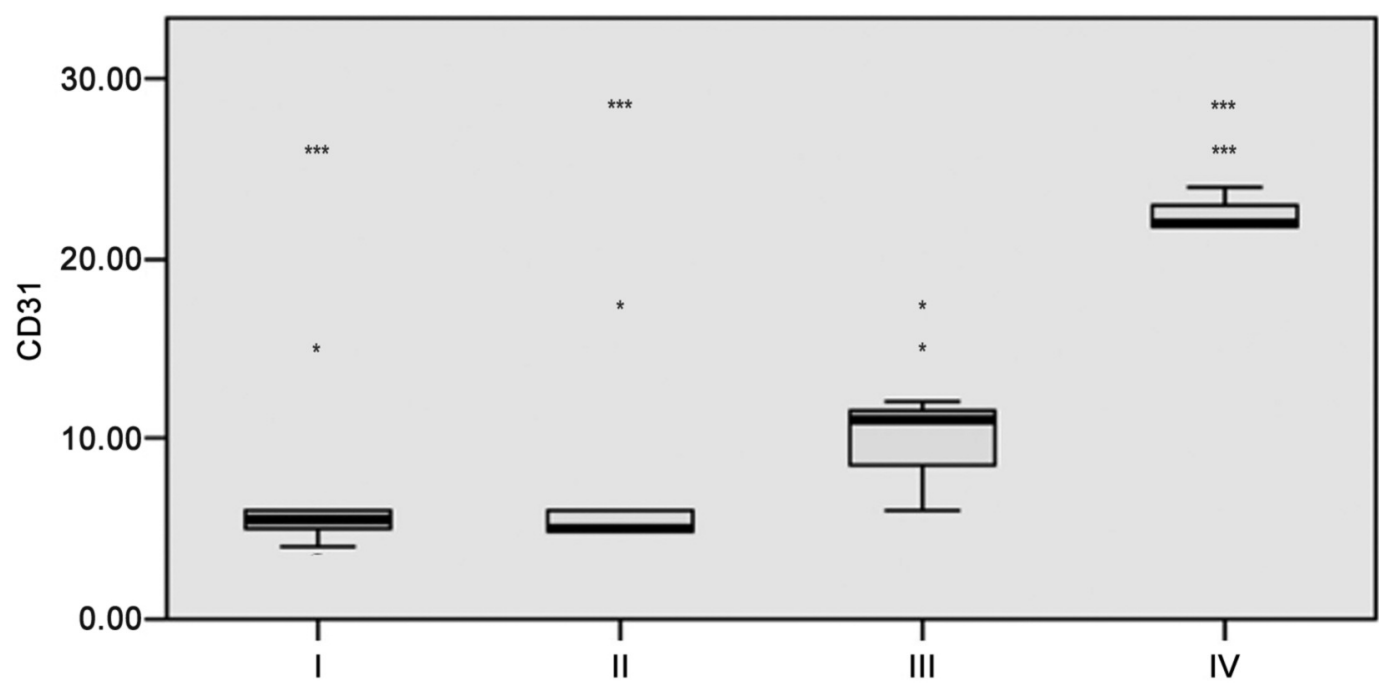

Figure 2. Microvascularization density in the four histological groups considered (Group I: scarce and peripheral lymphocytic infiltrate; group II: moderate and peripheral lymphocytic infiltrate; group III: abundant infiltrate at the periphery and in the center; group IV: diffuse lymphocytic infiltrate) $(* p<0.05 ; * * *<0.001)$.

the histological groups I and II. Cases with a higher VEGFA expression belonged mainly to the histological group III (a more advanced stage of the regressive process).

Immunohistochemical expression of VEGFR-2. VEGFR-2 was also expressed in the cytoplasm of tumour cells with a granular or diffuse pattern as well as in macrophages (positive internal control). VEGFR-2 expression was observed in most of the analysed cases (39/50 cases; $78 \%$ ), of which: i) 7 cases had a focal marker (5-25\% of the tumour cells), ii) 17 cases a multifocal marker and iii) 15 cases a diffuse marker. Regarding the intensity of the immunoreaction, i) 12 tumours (24\%) had a weak intensity, ii) 19 tumours $(38 \%)$ a moderate intensity and iii) 8 cases $(16 \%)$ a strong intensity. A diffuse, moderate marking on the epidermis was also observed (Figure 1C).

VEGFR-2 intensity was significantly associated with the mitotic index $(p=0.012)$. Regarding the stroma, tumours with abundant stroma were negative, whereas tumours of sparse and moderate stroma were mostly positive. This association was also statistically significant $(p=0.045)$.

VEGFR-2 positive cells were associated with the tumour group both for the extent of labelling $(p<0.001)$ as well as the intensity $(p=0.003)$. All tumours in group I expressed VEGFR2 with a multifocal $(n=12)$ or diffuse $(n=6)$ pattern, while group IV tumours were mostly negative for the receptor.

Immunohistochemical assessment of microvessel density $(M V D)$. The microvessel density, estimated by CD31 staining of endothelial cells (Figure 1D), showed an average of $9.08 \pm 6.58$ microvessels, a median of 6 , ranging from 2 to 28 vessels per $200 \times$ field. MVD varied in the different categories of the mitotic index $(p<0.001)$. Tumours with fewer mitoses had more microvessels and tumours with more mitoses had fewer microvessels. MVD also varied between the different stroma categories $(p=0.007)$, so that tumours with abundant stroma had more microvessels compared to tumours with scarce stroma.

MVD was lower in $\mathrm{CCH}$ from groups I (median=5.5, range $=2-10)$ and II (median=5.0, range $=3-6)$ and higher in groups III (median=11.0, range $=4-28$ ) and IV (median=22.0, range $=18-24$ ), with $p<0.001$. Considering the comparison between the different groups, we can see that the differences in MVD were significant between groups: i) I and III, ii) I and IV, iii) II and III, and iv) II and IV. There are no differences between groups I and II and groups III and IV (Figure 2).

\section{Discussion}

Tumour growth and metastasis are dependent, in part, on their capacity to induce the development of new blood vessels. In reverse, angiogenesis is one of the mechanisms that could has been involved in the spontaneous regression described in several tumours $(18,19)$.

Canine cutaneous histiocytoma, one of the most frequent tumours of the skin in young dogs, presents a rapid growth, however, after a few weeks its size decreases and the tumour mass regresses (20). In order to estimate angiogenesis in $\mathrm{CCH}$, we evaluated the expression of VEGF-A, VEGFR-2 and MVD in 50 tumours categorized in four histological 
groups, each corresponding to different stages of $\mathrm{CCH}$ regression. Our results indicate that angiogenesis, estimated by the angiogenic factor VEGF-A and its receptor, and by the CD31-positive microvessel density, seems to be a dynamic process along $\mathrm{CCH}$ regression. During early stages of canine histiocytoma development (groups I and II) there are only few blood vessels, most probably due to low levels of VEGF-A. In spite of tumour cells producing VEGFR-2 during these stages, since the signalization complex VEGFA/VEGFR-2 is a major regulator of blood vessel formation (21), the absence of VEGF-A can compromise $\mathrm{CCH}$ angiogenesis and tumoural growth. The scarcity of microvessels causes ischemia and cell hypoxia and could lead to the death of tumour cells by necrosis or apoptosis $(22,23)$. Considering the tumours in groups III and IV, which are more advanced with regards to the regression process, the abundant inflammatory response may be a stimulus that triggers the formation of microvessels through the production of angiogenic factors by the immune cells (24) and justify the greater number of vessels and higher VEGF expression in those tumours. There are also other circumstances that, theoretically, can contribute to angiogenesis in tumours in an advanced stage of the regression process, e.g., an increased infiltration of macrophages (10), or even hypoxia inducing a fast and strong increment in VEGF mRNA (25).

Our results are not similar to studies about angiogenesis in Langerhans cells human histiocytosis. These tumour cells express VEGF especially in disseminated lesions (26-28). This could be justified partially by the distinct clinic behavior and aggressiveness of these tumors when compared to canine cutaneous histiocytoma. Despite the similarities between human and canine histiocytosis, the human pathology in its disseminated form has a rapidly progressive multisystem involvement and an unfavorable prognosis (29). Canine cutaneous histiocytoma is usually unique, without a tendency for metastasis and with a favorable prognosis (1). Moreover, our results could emphasize the importance of VEGF as a therapeutic target during the early phases of malignancy in humans by inhibiting tumoral angiogenesis and early invasion of malignant cells $(26,28)$.

In conclusion, low expression of VEGF-A during early stages of HCC development, and consequently reduced microvessel density, may be crucial for initiating regression. The scarce production of VEGF in $\mathrm{CCH}$ by tumour cells does not justify the $\mathrm{CCH}$ regression, but the interaction of the tumour cells with the stroma and other systems, such as circulating immune cells, may contribute or even be the switch igniting the regression process.

\section{Conflicts of Interest}

The Authors declare no conflicts of interest with regard to this study.

\section{Authors' Contributions}

IP conceived and designed the study; DC, RF, IP and PR conducted the experiments, IP, JP, PR and FS analyzed the data; DC, RF, IP and FQ wrote the paper, IP, JP, FQ, FS discussed the manuscript.

\section{Acknowledgements}

The Authors would like to thank the financial support of project UIDB/00211/2020 funded by Fundação para a Ciência e Tecnologia/ Ministério da Ciência Tecnologia e Ensino Superior (FCT/MCTES) through national funds. Authors would also like to thank the support received by projects UIDB/04033/2020 and UIDB/CVT/00772/ 2020, from FCT/MCTES.

\section{References}

1 Moore PF: A review of histiocytic diseases of dogs and cats. Vet Pathol 51(1): 167-184, 2014. PMID: 24395976. DOI: 10.1177/ 0300985813510413

2 Paździor-Czapula K, Otrocka-Domagala I, Tadeusz R and Gesek M: Cytomorphometry of canine cutaneous histiocytoma. Pol J Vet Sci 17: 413-420, 2014. PMID: 25286647. DOI: 10.2478/pjvs-2014-0059

3 Paździor-Czapula K, Rotkiewicz T, Otrocka-Domagała I, Gesek $\mathrm{M}$ and Śmiech A: Morphology and immunophenotype of canine cutaneous histiocytic tumours with particular emphasis on diagnostic application. Vet Res Commun 39(1): 7-17, 2015. PMID: 25563490. DOI: 10.1007/s11259-014-9622-1

4 Pires I, Rodrigues P, Alves A, Queiroga FL, Silva F and Lopes C: Immunohistochemical and immunoelectron study of major histocompatibility complex class-ii antigen in canine cutaneous histiocytoma: Its relation to tumour regression. In Vivo 27(2): 257-262, 2013. PMID: 23422487.

5 Moore PF, Schrenzel MD, Affolter VK, Olivry T and Naydan D: Canine cutaneous histiocytoma is an epidermotropic langerhans cell histiocytosis that expresses cd1 and specific beta 2-integrin molecules. Am J Pathol 148(5): 1699-1708, 1996. PMID: 8623937.

6 Pires I, Queiroga FL, Alves A, Silva F and Lopes C: Decrease of e-cadherin expression in canine cutaneous histiocytoma appears to be related to its spontaneous regression. Anticancer Res 29(7): 2713-2717, 2009. PMID: 19596951.

7 Marchal T, Saint-Andre I, Magnol JP, Dezutter-Dambuyant C and Schmitt D: Ddendritic cells in dogs and cats: Models of study in human pathology. Pathol Biol (Paris) 43(10): 910-920, 1995. PMID: 8786898.

8 Aiba S, Rokugo M and Tagami H: Immunohistologic analysis of the phenomenon of spontaneous regression of numerous flat warts. Cancer 58(6): 1246-1251, 1986. PMID: 3017534. DOI: 10.1002/1097-0142(19860915)58:6<1246::aid-cncr2820580612> 3.0.co;2-e

9 Nicholls PK, Doorbar J, Moore RA, Peh W, Anderson DM and Stanley MA: Detection of viral DNA and e4 protein in basal keratinocytes of experimental canine oral papillomavirus lesions. Virology 284(1): 82-98, 2001. PMID: 11352670. DOI: 10.1006/ viro.2001.0868

10 Pires I, Alves A, Queiroga FL, Silva F and Lopes C: Regression of canine cutaneous histiocytoma: Reduced proliferation or increased apoptosis? Anticancer Res 33(4): 1397-1400, 2013. PMID: 23564778. 
11 Kipar A, Baumgärtner W, Kremmer E, Frese K and Weiss E: Expression of major histocompatibility complex class ii antigen in neoplastic cells of canine cutaneous histiocytoma. Vet Immunol Immunopathol 62(1): 1-13, 1998. PMID: 9618864. DOI: $10.1016 / \mathrm{s} 0165-2427(97) 00170-0$

12 Kaim U, Moritz A, Failing $\mathrm{K}$ and Baumgärtner W: The regression of a canine langerhans cell tumour is associated with increased expression of il-2, tnf- $\alpha$, ifn- $\gamma$ and inos mrna. Immunology 118(4): 472-482, 2006. PMID: 16764690. DOI: 10.1111/j.1365-2567.2006.02394.x

13 Puff C, Risha E and Baumgärtner W: Regression of canine cutaneous histiocytoma is associated with an orchestrated expression of matrix metalloproteinases. J Comp Pathol 149(23): 208-215, 2013. PMID: 23582970. DOI: 10.1016/j.jcpa. 2013.01.014

14 Potente M, Gerhardt $\mathrm{H}$ and Carmeliet P: Basic and therapeutic aspects of angiogenesis. Cell 146(6): 873-887, 2011. PMID: 21925313. DOI: 10.1016/j.cell.2011.08.039

15 de Brot S, Ntekim A, Cardenas R, James V, Allegrucci C, Heery DM, Bates DO, Ødum N, Persson JL and Mongan NP: Regulation of vascular endothelial growth factor in prostate cancer. Endocr Relat Cancer 22(3): R107-R123, 2015. PMID: 25870249. DOI: $10.1530 /$ ERC-15-0123

16 Carvalho MI, Silva-Carvalho R, Pires I, Prada J, Bianchini R, Jensen-Jarolin E and Queiroga FL: A comparative approach of tumour-associated inflammation in mammary cancer between humans and dogs. Biomed Res Int 2016: 4917387, 2016. PMID: 28053982. DOI: $10.1155 / 2016 / 4917387$

17 Cockerell GL and Slauson DO: Patterns of lymphoid infiltrate in the canine cutaneous histiocytoma. J Comp Pathol 89(2): 193203, 1979. PMID: 222817. DOI: 10.1016/0021-9975(79)900586

18 Salman T: Spontaneous tumor regression. J Oncol Sci 2(1): 1-4, 2016. DOI: $10.1016 /$ j.jons.2016.04.008

19 Ghim S-j, Newsome J, Bell J, Sundberg JP, Schlegel R and Jenson AB: Spontaneously regressing oral papillomas induce systemic antibodies that neutralize canine oral papillomavirus. Exp Mol Pathol 68(3): 147-151, 2000. PMID: 10816383. DOI: 10.1006/exmp.1999.2298

20 Pires ICR: Histiocitoma cutâneo canino: Estudo biomorfológico da regressão, 2004.

21 Nakayama $\mathrm{M}$ and Berger P: Coordination of vegf receptor trafficking and signaling by coreceptors. Exp Cell Res 319(9): 1340-1347, 2013. PMID: 23499743. DOI: 10.1016/j.yexcr. 2013.03.008
22 Hou W, Zhang Q, Yan Z, Chen R, Zeh Iii HJ, Kang R, Lotze MT and Tang D: Strange attractors: Damps and autophagy link tumour cell death and immunity. Cell Death Dis 4(e966), 2013. PMID: 24336086. DOI: 10.1038 /cddis.2013.493

23 van Noesel MM and Versteeg R: Pediatric neuroblastomas: Genetic and epigenetic 'danse macabre'. Gene 325: 1-15, 2004. PMID: 14697505. DOI: 10.1016/j.gene.2003.09.042

24 Raposo TP, Pires I, Carvalho MI, Prada J, Argyle DJ, Queiroga FL: Tumour-associated macrophages are associated with vascular endothelial growth factor expression in canine mammary tumours. Vet Comp Oncol 13(4): 464-74, 2015. PMID: 24119241. DOI: 10.1111/vco.12067

25 Veikkola T, Karkkainen M, Claesson-Welsh L and Alitalo K: Regulation of angiogenesis via vascular endothelial growth factor receptors. Cancer Res 60(2): 203-212, 2000. PMID: 10667560.

26 Mehrazma M, Mahjoob F, Rafsanjani KA, Jafari D, Farahani SJ and Lahouti A: Vegf and p53 biomarkers in pediatric patients with langerhans cell histiocytosis. J Pediatr Hematol Oncol 35(7): e292-e295, 2013. PMID: 23619110. DOI: 10.1097/MPH. $0 \mathrm{~b} 013 \mathrm{e} 31828 \mathrm{e} 51 \mathrm{ae}$

27 Pavlakovic H, Von Schütz V, Rössler J, Koscielniak E, Havers $\mathrm{W}$ and Schweigerer L: Quantification of angiogenesis stimulators in children with solid malignancies. Int $\mathrm{J}$ Cancer 92(5): 756-760, 2001. PMID: 11340583. DOI: 10.1002/10970215(20010601)92:5<756::aid-ijc1253>3.0.co;2-f

28 Dina A, Zahava V and Iness M: The role of vascular endothelial growth factor in Langerhans cell histiocytosis. J Pediatr Hematol Oncol 27(2): 62-66, 2005. PMID: 15701978. DOI: 10.1097/ 01.mph.0000154069.81149.5f

29 Braier J, Chantada G, Rosso D, Bernaldez P, Amaral D, Latella A, Balancini B, Masautis A and Goldberg J: Langerhans cell histiocytosis: retrospective evaluation of 123 patients at a single institution. J Pediatr Hematol Oncol 16(5): 337-385, 1999. PMID: 10505313. DOI: 10.1080/088800199276921

Received August 7, 2020

Revised September 6, 2020 Accepted September 16, 2020 